\title{
Light-Activated Nanoscale Gas Vesicles Selectively Kill Tumor Cells
}

\author{
Ann Fernando ${ }^{\mathrm{a}, \mathrm{c}}$ and Jean Gariépy* ${ }^{\mathrm{a}, \mathrm{b}, \mathrm{c}}$ \\ a Department of Pharmaceutical Sciences, The University of Toronto, 27 King's College \\ Circle Toronto, Ontario M5S 1A1 Canada. ann.fernando@mail.utoronto.ca, \\ jean.gariepy@utoronto.ca. \\ ${ }^{\mathrm{b}}$ Department of Medical Biophysics, The University of Toronto, 27 King's College Circle \\ Toronto, Ontario M5S 1A1 Canada \\ ${ }^{\mathrm{c}}$ Sunnybrook Research Institute, 2075 Bayview Ave, Toronto, ON M4N 3M5
}




\begin{abstract}
Protein-based nanobubbles such as halophilic archaeabacterial gas vesicles (GVs) represent a new class of stable, homogeneous nanoparticles with acoustic properties that allow them to be visualized by ultrasound (US) waves. To design GVs as theranostic agents, we modified them to respond to light, with a view to locally generate reactive oxygen species that can kill cancer cells. Specifically, up to 60,000 photoreactive chlorin $e 6(\mathrm{Ce} 6)$ molecules were chemically attached to lysine $\varepsilon$-amino groups present on the surface of each purified Halobacterium sp. NRC-1 GV. The resulting fluorescent NRC-1 Ce6-GVs have dimensions comparable to that of native GVs and were efficiently taken up by human breast [MCF-7] and human hypopharyngeal [FaDu-GFP] cancer cells as monitored by confocal microscopy and flow cytometry. When exposed to light, internalized Ce6-GVs were 200-fold more effective on a molar basis than free Ce6 at killing cells. These results demonstrate the potential of $\mathrm{Ce} 6-\mathrm{GVs}$ as novel and promising nanomaterials for image-guided photodynamic therapy.
\end{abstract}

Keywords: Cancer; Chlorin e6; Gas Vesicles; Nanobubbles; Photodynamic therapy 


\section{Introduction}

Image-guided therapies are precision medicine-based treatments being developed in many cases to treat cancer patients. These therapies benefit from the development of theranostic agents that integrate both targeted diagnostic and therapeutic functions. ${ }^{1,2,3}$

Theranostics often incorporate radionuclides or MR contrast agents as imaging probes in conjunction with cytotoxic nuclides or drug-conjugates linked to a tumor-targeting agent such as a small ligand or an antibody that delivers the payload. An alternate design strategy to reduce potential off-target toxicities arising from delivering these agents systemically is to create theranostics that are only activated within the tumor environment (pro-drug like theranostics that become activated as a result of a local change in $\mathrm{pH}$ or by tumor-associated proteases) or upon exposing tumors to an external, focused energy source that would remotely activate such agents within the tumor environment only. Nanoparticles are particularly suited to this purpose as they tend to accumulate within the abnormal tumor neovasculature as a consequence of the enhanced permeability and retention (EPR) effect. ${ }^{4,5}$ Tumor selectivity can be encoded into nanoparticles by making them sensitive to an externally applied, ultrasound and light source; physical methods that are presently being used in image-guided therapy. Protein-based gas vesicles (GVs) represent an example of a naturally occurring nanostructure that could be used for this purpose. In the present study, we focused on GV nanostructures genetically encoded by the aquatic halophilic archeon Halobacterium salinarum NRC-1. ${ }^{6}$ They are composed of two dominant subunits termed gas vesicle protein A (GvpA) and gas vesicle protein $\mathrm{C}$ (GvpC). ${ }^{6}$ These GVs have recently been shown to respond to ultrasound waves and to produce hyperpolarized magnetic resonance contrast for imaging. ${ }^{7,8}$ In the present study, we explored the potential of such GVs as photodynamic therapy (PDT) agents. We report the design of a light-activated GV nanoparticle where the photo-reactive dye chlorin $e 6$ (Ce6) was coupled to free amino groups on the surface of GVs. The resulting nanoscale size structures were found to readily accumulate into cancer cells and kill them in response to light activation displaying remarkable enhancements in cytotoxicity towards cancer cells relative to free $\mathrm{C} e 6$. 


\section{Results and Discussion}

\section{Preparation and characterization of GVs modified with chlorin e6 (Ce6-GVs)}

Gas vesicles (GVs) are air-filled nanobubbles that have been shown to respond to ultrasound (US) waves. ${ }^{7}$ In this study, GVs were isolated from the Halobacterium sp. NRC-1 to serve as nanocarriers for the light-activated drug, chlorin $e 6$ (Ce6) with a view of creating therapeutic GVs beyond their US-imaging properties. As such, NRC-1 GVs were purified and their surface modified with $\mathrm{Ce} 6$ to demonstrate their ability to be internalized by cancer cells and to enhance the cytotoxicity of $\mathrm{Ce} 6$ as a photodynamic therapy (PDT) agent. Ce6-modified GVs represent a first attempt at generating GV-based theranostic agents aimed at killing cancer cells. Specifically, Ce6 absorbs light with maxima observed at $400 \mathrm{~nm}$ and $660 \mathrm{~nm}$. The photodynamic effect occurs upon absorption in the red region of the spectrum where human tissue is optically transparent, reaching tissue depth of up to $16 \mathrm{~mm} .{ }^{9,10} \mathrm{Ce} 6$ is also a fluorescent compound, which enables the use of optical imaging to detect its accumulation into tumors. ${ }^{11}$

Halobacterium sp. NRC-1 GVs used in this study are composed of two major protein species (GvpA and GvpC) as well as several minor proteins involved in the nanobubble assembly. Importantly, GvpA accounts for $>90 \%$ of the nanobubble structure and shell ${ }^{6}$. GvpC binds non-covalently to the surface of the GV shell. However, it is stripped from the nanobubble surface during the process of isolating GVs from the halobacterium, as confirmed by mass spectrometry of our GV preparations (Supplementary Figure S1). As such, the two lysine $\varepsilon$-amino groups present in GvpA (lysine 19 [helix I] and lysine 60 [helix II] of GvpA; depicted in Fig. 1A) represent the main sites for covalently attaching Ce6 to the surface of GVs.

Experimentally, wild type gas vesicles (WT GV) from the Halobacterium sp. NRC-1 were isolated by flotation from lysed halobacteria and purified by repeated centrifugation steps as described elsewhere (Fig. 1B). ${ }^{12}$ The carboxylic groups of chlorin $e 6$ were then activated with EDAC and sulfo-NHS to form esters that reacted with the two free $\varepsilon$ amino groups of GvpA on WT GVs, resulting in an olive-green colored nanobubble preparation termed Ce6-GVs (Fig. 1B).

The particle size of these GVs was studied by dynamic light scattering (DLS) and transmission electron microscopy (TEM). Both WT GVs and Ce6-GVs adopted a characteristic prolate-spheroid, lemon-shaped structure of comparable dimensions as confirmed from electron micrographs, suggesting that decorating the surface of GVs with Ce6 molecules minimally affected particle structure and size (Fig. 2, Table 1). The length of Ce6-GVs along their long and short axes were measured to be $417 \mathrm{~nm}$ and 196 $\mathrm{nm}$ respectively according to TEM (Fig. 2, Table 1). Estimates of WT GVs and Ce6-GVs particle diameter by DLS $(273 \pm 3 \mathrm{~nm}$ and $338 \pm 39 \mathrm{~nm}$ respectively; Table 1) assumes that GVs are spherical. The calculated diameters approximate the average length of both their short and long axes as determined by TEM (Table 1). The zeta potential of Ce6-GVs was significantly lower $(-30 \mathrm{mV})$ than WT GVs $(-2.4 \mathrm{mV})$ as Ce6 contains three negatively charged carboxylic acids (Table 1). The increased negative charge suggests that Ce6-GVs may have good colloidal stability due to repulsion between the nanoparticles. 
Using surface area measurements of WT GVs based on TEM micrographs (Fig. 2 A,B; Table 1; $380 \mathrm{~nm}$ by $260 \mathrm{~nm}$ ) along with the experimentally determined surface area of one GvpA molecule, the main repeating structural component of the nanobubble shell, ${ }^{13}$ the mass of one Halobacterium GV was estimated to be $444 \mathrm{MDa}$ (See Supplementary Figure S2) with a single NRC-1 GV being composed of $~ 55,000$ GvpA proteins. Since the two lysines present in GvpA (K19 in helix I and K60 in helix II; Fig. 1A) are projected to be solvent-accessible based on solid-state NMR, ${ }^{14}$ one can project that up to $110,000 \varepsilon$-amino groups are available to react with an amine reactive form of Ce6. To accurately determine the extent of $\mathrm{Ce} 6$ conjugated to WT GVs, we measured the total amount of protein (amino acid analysis) and released $\mathrm{Ce} 6$ content by hydrolyzing Ce6GVs samples in $6 \mathrm{~N} \mathrm{HCl}$. It was estimated from hydrolysates that $\sim 60,000$ molecules of Ce6 molecules were coupled per nanobubble; a value representing a loading efficiency of approximately one Ce6 molecule per GypA protein.

The presence of $\mathrm{Ce} 6$ on GVs was further confirmed by recording the fluorescence emission spectra of $\mathrm{Ce} 6$ released by acid hydrolysis from Ce6-GVs (Fig.3). The observed fluorescence emission maximum at $660 \mathrm{~nm}$ recorded for Ce6-GVs was comparable to that of free $\mathrm{Ce} 6$, while hydrolysates from WT GVs were non-fluorescent (Fig. 3). This finding is comparable to the published fluorescence emission spectra of equimolar doses of Ce6-liposomes and free $\mathrm{Ce}^{15}$ or $\mathrm{Ce} 6$-albumin nanoparticle formulations. ${ }^{16}$ These results indicate that $\mathrm{Ce} 6$ can be efficiently loaded onto GVs and also demonstrate that conjugation to GVs does not significantly alter the spectral properties of $\mathrm{Ce}$, nor the shape and dimensions of GVs. 


\section{Ce6-GVs are taken up by cancer cells and accumulate in endosomes}

The light-induced cytotoxicity of Ce6-containing GVs depends on their ability to be internalized by cancer cells. Upon reaching the cytoplasm and following light activation, Ce6 molecules are excited to their singlet $\rightarrow$ triplet states where their energy can be transferred to $\mathrm{O}_{2}$ to generate reactive singlet oxygen $\left({ }^{1} \mathrm{O}_{2}\right) \cdot{ }^{17}$ Alternatively, activated $\mathrm{Ce} 6$ can react directly with proteins, nucleic acids and lipids to form reactive oxygen species (ROS; superoxide anion, hydroxyl radical, hydrogen peroxide) causing oxidative damage leading to cell death. ${ }^{17,18}$

The cellular uptake of Ce6-GVs, WT GVs and free Ce6 by human breast carcinoma MCF-7 and human hypopharyngeal squamous cell carcinoma FaDu cells was thus assessed by flow cytometry and confocal microscopy. For both techniques, fluorescence emission signals arising from the chlorin $e 6$ chromophore $\left(\lambda_{\mathrm{exc}} 403 \mathrm{~nm}\right)$ were captured between $660-680 \mathrm{~nm}$ (Fig. 3). The intracellular uptake of Ce6-GVs and free Ce6 was monitored by flow cytometry in terms of mean fluorescence intensities (MFI) as a function of time and temperature (Fig. 4). As expected, cellular uptake did not take place at $4{ }^{\circ} \mathrm{C}$ for either cell lines (Fig. 4 A,C) while the internalization of Ce6-GVs at $37{ }^{\circ} \mathrm{C}$ reached a plateau at 8 hours and 22 hours for the FaDu-GFP and MCF-7 cell lines respectively (Fig.4 B,D). In contrast, free Ce6 minimally enters these cells while no fluorescence signal could be detected for WT GVs (Fig. 4). These results show that conjugation of Ce6 to GVs improves the intracellular delivery of this drug into cancer cells. Cellular uptake of other $\mathrm{Ce} 6$ nanoparticles have been previously described as exemplified by Ce6-octalysine conjugated to superparamagnetic iron oxide nanoparticles being taken up by SKOV3 cells relative to free Ce6. ${ }^{19}$

The cellular uptake of Ce6-GVs at $37^{\circ} \mathrm{C}$ into MCF-7 and FaDu-GFP cells was further confirmed by confocal microscopy (Fig. 5). Specifically, a punctate pattern of Ce6 fluorescence (in red) is observed in the cytosol of these cells after an 8-hour incubation period suggesting their compartmentalization into organelles such as endosomes or vacuoles. Ce6-GVs did not reach the cell nucleus (Hoescht dye; blue color nuclei; Fig. 5 $\mathrm{A}, \mathrm{E})$. This cytoplasmic distribution pattern has been observed for other Ce6-conjugates and nanoparticles. 9,20 


\section{Gas Vesicles dramatically enhance the phototoxicity of Ce6 towards cancer cells}

Human MCF-7 breast cancer and FaDu-GFP pharyngeal cancer cells were exposed to increasing concentrations of $\mathrm{Ce} 6-\mathrm{GVs}$, free $\mathrm{C} e 6$, or an equivalent amount of WT GVs for 24 hours, followed by a 10-minute exposure to light. Their viability was assessed 24hours later and reported as a function of the molar concentration of Ce6 being given (Fig. 6). Both cell lines remain viable in the absence of light treatment except for cells exposed to high doses of Ce6-GVs $\left(10^{-5} \mathrm{M}\right.$ range; Fig $\left.6 \mathrm{~A}, \mathrm{C}\right)$. Upon light activation, cell viability was lost in a dose-dependent manner with $\mathrm{C} e 6-\mathrm{GV}$ being more potent than the free drug (Fig.6, B,D). Specifically, $\mathrm{CD}_{50}$ values towards both cell lines were determined for Ce6-GV and free Ce6 (Table 2) following light exposure, with the drug covalently attached to GVs being 200-fold more toxic on a drug molar basis than free Ce6 (Fig. 6 B,D). WT GVs were only toxic towards MCF-7 or FaDu-GFP at very high concentrations (at concentrations $>10^{-4} \mathrm{M}$ relative to comparable $\mathrm{C} e 6-\mathrm{GV}$ doses given; Supplementary Figure S3) The enhanced toxicity observed for Ce6-GV correlates with its greater cellular uptake seen in both cell lines in contrast to free Ce6 (Fig. 4,5). This enhancement in light-activated toxicity relative to the free form of the drug has been observed in SKOV3 and MDA-MB-231 cells for other Ce6-nanoparticles such as Ce6SPION ${ }^{19}$ or Ce6-conjugated poly(ethylene glycol)-poly-(d,l-lactide) ${ }^{21}$ nanoparticles, likely owing to their improved cellular uptake. In contrast, the photosensitizing efficacy and toxicity of a $\mathrm{Ce} 6$ conjugated human serum albumin nanoparticle was comparable to that of free $\mathrm{Ce}^{22}$ suggesting that all Ce6-nanoparticle formulations are not equivalent in terms of enhanced cytotoxicity. Interestingly, agents such as $\mathrm{Ce} 6$ can also be activated by ultrasound waves; an approach termed sonodynamic therapy that may allow one to target deep-seated tumors following the activation of the photosensitizer agent. ${ }^{23}$ For example, the viability of $\mathrm{H} 22$ hepatocellular carcinoma cells exposed to $50 \mu \mathrm{g} / \mathrm{ml}$ of free Ce6 for 4 hours was decreased by $40 \%$ following ultrasound treatment. ${ }^{24}$ Although ultrasound is a less potent activation modality than light, our present study now suggests that the use of Ce6-GVs rather than free Ce6 may address this limitation. As such, Ce6$\mathrm{GVs}$ are now being assessed as a new class of sono-sensitive agents. 


\section{The enhanced toxicity of Ce6-GVs towards cells is mechanistically related to their cellular accumulation and the production of intracellular ROS.}

The phototoxic effect of $\mathrm{Ce} 6$ is related to the production of intracellular reactive oxygen species (ROS) as a consequence of the drug being exposed to light. This effect was measured by treating cells with the green fluorescence-emitting probe, DCFH-DA, prior to illumination. As presented in Fig. 7, the DCFH-DA fluorescence emission signal for MCF-7 and FaDu-GFP treated with Ce6-GV confirm the presence of ROS in light-treated cells only (Fig 7A,B). For MCF-7 and FaDu-GFP cell lines, significantly more ROS were produced when $\mathrm{Ce} 6-\mathrm{GV}$-loaded cells were exposed to light as compared to Ce6-GVloaded cells kept in the dark ( $\mathrm{p}=0.0052$ and $\mathrm{p}=0.0004$ respectively). The difference in ROS production due to $\mathrm{Ce} 6 \mathrm{-GV}$ treatment was greater than ROS production due to free Ce6 treatment in either MCF-7 $(\mathrm{p}=0.0385)$ or FaDu-GFP cell lines $(\mathrm{p}=0.0126)$. These results confirm that conjugating $\mathrm{Ce} 6$ to $\mathrm{GVs}$ does not impair their capacity to produce ROS and is consistent with the superior uptake of Ce6-GV relative to free Ce6 demonstrated by flow cytometry (Fig. 4). Internalization events favoring the accumulation of Ce6-GVs over the free drug inside cells probably play a dominant role in enhancing the toxicity of Ce6 towards cancer cells. The generation of ROS is expected to cause damage to intracellular components such as protein, DNA, or membranes and trigger apoptotic or necrotic pathways to mediate cell death. ${ }^{25}$ In the case of Ce6-GVs, confocal images presented in Fig.5 suggest that these nanobubbles never reach the cell nucleus indicating that the generated reactive oxygen species following illumination are mainly deposited in the cytoplasm of these cells, preferentially causing the oxidation of lipids and proteins rather than damaging nucleic acid species. ${ }^{26,27}$

\section{Conclusions}

We have covalently linked the photoreactive drug Ce6 to nanoscale gas vesicles (GVs) with a view to expand their potential as nanoparticles responding to ultrasound waves and now to light. This newly-introduced function suggests that GVs represent a good platform for designing stable theranostic agents that can be externally activated to serve as both imaging and now as locally-activated therapeutic agents. Specifically, Ce6-GVs were efficiently internalized by MCF-7 and FaDu-GFP cancer cells and were highly effective in killing them in vitro upon light activation, relative to the free drug. 


\section{Material and Methods}

\section{Production and Purification of Gas Vesicles}

Gas vesicles were isolated from Halobacterium sp. NRC-1. Cells were cultured in $\mathrm{CM}+$ growth medium [4.3M Sodium Chloride, $81 \mathrm{mM}$ Magnesium sulfate heptahydrate, 10 $\mathrm{mM}$ trisodium citrate dehydrate, $27 \mathrm{mM}$ potassium chloride, $0.5 \%$ casein hydrolysate (Sigma), $0.3 \%$ yeast extract (Difco)] at $42{ }^{\circ} \mathrm{C}$, shaking at $100 \mathrm{rpm}$ for one week until cells are confluent. The cultures were subsequently transferred to sterile separatory funnels and the fraction of cells expressing GVs at a high level were allowed to accumulate at the top by flotation over a one-week period. GVs were collected by hypotonic lysis of the buoyant cell fraction using $1 \mathrm{mM} \mathrm{MgSO} 4$ solution. After lysis, GVs were purified using repeated centrifugation steps at $300 \mathrm{~g}$ overnight for at least three days and washed with PBS at each step. Intact buoyant vesicles were re-suspended in PBS and dialyzed against PBS (100 kDa MWCO dialysis membrane; Biotech CE) as a final step.

\section{Fabrication of chlorin e6-decorated GVs}

Chlorin $e 6$ (Ce6; $119 \mu \mathrm{g}$, Cayman Chemicals, 20mM), 1- 1-(3-di-methylaminopropyl)-3ethylcarbodiimide hydrochloride (EDAC) (116 $\mu \mathrm{g}, 60 \mathrm{mM})$, and sulfo-Nhydroxysuccinimide (sulfo-NHS) $(132 \mu \mathrm{g}, 60 \mathrm{mM})$ in a volume of $10 \mu \mathrm{L}$ DMF was rotated for 3 hours at $25{ }^{\circ} \mathrm{C}$ in the dark, in order to activate the three carboxyl groups of Ce6. The amount of $\mathrm{Ce} 6$ used corresponded to a 1000-fold molar excess of this agent relative to the available amino groups present in $800 \mu \mathrm{g}$ of GVs. Separately, $50 \mu \mathrm{L}$ of DMF was added to $800 \mu \mathrm{g}$ of GVs suspended in $60 \mu \mathrm{L}$ of PBS at $\mathrm{pH} 7$. The activated Ce6 solution $(10 \mu \mathrm{L})$ was then added to the $\mathrm{GV}$ suspension and the final mixture was rotated for 5 hours in the dark at $25^{\circ} \mathrm{C}$. The nanoparticles were subsequently separated by centrifugation $\left(300 \mathrm{~g}, 25{ }^{\circ} \mathrm{C}\right)$ and washed with PBS to remove residual free Ce6. The grey-colored, Ce6-modified GV suspension was stored at $4{ }^{\circ} \mathrm{C}$ in PBS.

\section{Determination of $\mathrm{Ce} 6$ loading on GVs}

The amino acid composition and protein concentration of the Ce6-GV preparation were accurately determined by amino acid hydrolysis (Hospital For Sick Children Toronto, $\mathrm{CA})$. Ce6-GV samples were also hydrolyzed $\left(110^{\circ} \mathrm{C}\right.$ in $6 \mathrm{~N} \mathrm{HCl}$ in vacuo) to release $\mathrm{Ce} 6$ covalently bound to lysine $\varepsilon$-amino groups on the surface of GVs. The concentration of Ce6 released from Ce6-GV sample hydrolysates was determined by fluorescence spectroscopy using a free Ce6 standard curve $\left(\lambda_{\text {exc }} 400 \mathrm{~nm} ; \lambda_{\text {em }} 660 \mathrm{~nm}\right.$; see Supplementary Figure S4). 


\section{Nanoparticle Characterization}

The shape and size of intact Ce6-GVs were established by transmission electron microscopy (Phillips/FEI Tecnai Hillsboro OR) and by dynamic light scattering (Zetasizer Nano ZS, Malvern, UK). The size distribution of Ce6-GVs derived from electron micrographs was estimated using ImageJ software. ${ }^{28}$ Emission spectra of Ce6GVs were recorded using a fluorescence microplate reader (Synergy H1) using a concentration of $1.9 \mu \mathrm{M}$ of $\mathrm{Ce} 6$ in the form of free $\mathrm{Ce} 6$ or $\mathrm{Ce} 6-\mathrm{GV}$ or the equivalent amount of WT GV. The Zeta potential of Ce6-GV or WT GV nanoparticles (final concentration of $40 \mathrm{pM}$ in $990 \mu \mathrm{L}$ of distilled water) was determined using the Zetasizer Nano ZS (Malvern, UK).

\section{Cellular uptake studies}

For cellular studies, the human breast cancer cell line MCF-7 was purchased from ATCC (cat.\# HTB-22). The human hypopharyngeal cancer cell line FaDu-GFP (AntiCancer Inc., San Diego, CA) was a gift from Dr. David Goertz (Sunnybrook Research Institute, Toronto). MCF-7 and FaDu-GFP cells ( $10^{6}$ cells) were seeded into wells of 12 -well plates and incubated overnight $\left(37{ }^{\circ} \mathrm{C}, 5 \% \mathrm{CO} 2\right)$ to enable cell attachment. Ce6-GVs, free $\mathrm{Ce} 6$ (both adjusted to $670 \mathrm{nM} \mathrm{Ce6)}$ ) or native GVs (equivalent to the Ce6-GV concentration) were dispensed into wells. Plates were then kept rotating at $4{ }^{\circ} \mathrm{C}$ or $37{ }^{\circ} \mathrm{C}$ in an incubator with cells being collected over time [0.5 to 48 hours] to assess their viability and the internalization of $\mathrm{Ce} 6$ and GVs. Briefly, cells were trypsinized and washed with PBS once. Cellular uptake of $\mathrm{Ce} 6$ as a function of incubation time was measured by flow cytometry as mean fluorescence intensities (MFI); $\square_{\text {exc }} 405 \mathrm{~nm}, \square$ P Filter 660-2 nm, Dichroic 635LP; LSR II, BD Biosciences, San Jose, CA). Internalization events of Ce6 and Ce6-GVs in MCF-7 or FaDu-GFP cells were also recorded by confocal microscopy. Specifically, MCF-7 or FaDu-GFP cells were seeded into an 8-well confocal chamber at a density of $10^{5}$ cells for $24 \mathrm{~h}\left(37^{\circ} \mathrm{C}, 5 \% \mathrm{CO}_{2}\right)$. The cells were then treated with $\mathrm{Ce} 6-\mathrm{GV}$, free $\mathrm{Ce} 6$, or an equivalent amount of WT GV for 8 hours. Cell nuclei were stained using the cell permeable dye Hoescht 33342 ( $1 \mu \mathrm{M}$; ThermoFischer). Cells were then washed with PBS and both phase contrast and fluorescence ( $\square_{\text {exc }} 403 \mathrm{~nm}, \square_{\mathrm{em}} 663-738 \mathrm{~nm}$ ) images captured using a Nikon A1 laser-scanning microscope. 


\section{Cell viability measurements}

The cytotoxicity of Ce6-GV, free Ce6, or WT GV towards MCF-7 human breast cancer and FaDu-GFP pharyngeal cancer cell lines was assessed using the tetrazolium salt-based WST-1 cell proliferation assay. Briefly, cells were seeded at an initial density of $10^{5}$ cells and were incubated overnight $\left(37{ }^{\circ} \mathrm{C}, 5 \% \mathrm{CO}_{2}\right)$ to enable attachment. The next day, cells were treated for 24 hours with serial dilutions of either Ce6-GVs, equivalent molar doses of free $\mathrm{Ce} 6$, or corresponding amounts of native GVs relative to Ce6-GVs. Cells were subsequently washed once with PBS and exposed to a LED light source $(660 \mathrm{~nm})$ for 10 min (ABI 25W Deep Red). The source irradiance was determined to be $15-35 \mathrm{~mW} / \mathrm{cm}^{2}$ (Newport Powermeter 1918-R). The plate containing Ce6-GV, free Ce6, or WT GVtreated cells not exposed to the light source served as a control for non-light associated cytotoxicities. Following light exposure, cells were incubated for an additional 24 hours in the dark. After this period, media was discarded and cells were incubated with $10 \mu \mathrm{L}$ of WST-1 reagent (Roche) and $90 \mu \mathrm{L}$ of growth medium for 4 hours. Absorbance readings at $480 \mathrm{~nm}$ were then recorded using a microplate reader (Synergy H1). Cell viability was normalized relative to a positive control $(50 \mu \mathrm{g} / \mathrm{mL}$ Doxorubicin- $\mathrm{HCl}$ treatment leading to complete cell death) and a negative control (untreated cells) using the following equation:

Equation 1. ((Absorbance sample $_{-}-$Absorbance $\left._{\text {positive control }}\right) /$ (Absorbance $_{\text {negative }}$ control - Absorbance positive control) $)$ X 100\%

Two independent trials were performed and each data point represents the average cell survival percentage $( \pm \mathrm{SD})$ derived from proliferation assays performed in triplicate for each tested treatment. 


\section{Detection of intracellular reactive oxygen species (ROS)}

2,7-Dichlorofluorescein diacetate (DCF-DA) (Sigma) is a non-fluorescent, cellpermeable probe used to measure intracellular ROS. DCF-DA is deacetylated by intracellular enzymes to a non-fluorescent dye that is finally oxidized by ROS to the fluorescent compound dichlorofluorescein. ${ }^{29}$ For this assay, MCF-7 or FaDu-GFP cells were seeded into 24 -well plates ( $5 \times 10^{5}$ cells) overnight and were subsequently treated for 8 hours with $\mathrm{C} e 6-\mathrm{GV}$, free $\mathrm{C} e 6$ (based on a molar concentration equivalent of 500 $\mathrm{nM}$ ) or WT GV (at a dose identical to that of Ce6-GV) to allow for their internalization. Cells were then washed with PBS and the plates were either exposed to light $(660 \mathrm{~nm})$ for $10 \mathrm{~min}$ or kept in the dark. After the light treatment, $2.5 \mu \mathrm{l}$ of $1 \mathrm{mM}$ DCF-DA dissolved in DMSO ( $5 \mu \mathrm{M}$ final concentration in wells) was dispensed into each well and left for 30 min at $37{ }^{\circ} \mathrm{C}$ in an incubator. Cells were washed twice under dark conditions with PBS and the DCF fluorescence emission signal was recorded using a micro-plate reader ( $\lambda_{\text {exc }}$ $488 \mathrm{~nm} ; \lambda_{\mathrm{em}} 525 \mathrm{~nm}$ ) (Synergy H1). The fluorescence emission values were normalized relative to the average DCF fluorescence of cells treated with free Ce6 in the absence of light. The results were analyzed with a one-tailed paired t-test to evaluate the difference between paired groups based on sample size of $n=10$. Significant differences were determined as $\alpha<0.05$. Normality of the data distribution was confirmed using Kolmogorov-Smirnov test.

\section{Data Availability}

Data is available upon reasonable request from the corresponding author.

\section{References}

1. Warner, S. Diagnostics + Therapy = Theranostics. [online] The Scientist Magazine®. Available at: https://www.the-scientist.com/profession/diagnostics-therapy-theranostics49660 (2019) [Accessed 26 Apr. 2019].

2. Taber, S.W., Fingar, V.H., Coots, C.T., Wieman, T.J. Photodynamic Therapy Using Mono-L-Aspartyl Chlorin E6 (Npe6) for The Treatment Of Cutaneous Disease: A Phase I Clinical Study. Clin. Cancer Res. 4, 2741-2746 (1998).

3. Baum, R.P., Kulkarni, H.R. Theranostics: From Molecular Imaging Using Ga-68 Labeled Tracers And PET/CT To Personalized Radionuclide Therapy-The Bad Berka Experience." Theranostics. 2, 437-447 (2012).

4. Matsumura, Y., Maeda, H. A New Concept For Macromolecular Therapeutics In Chemotherapy: Mechanism Of Tumoritrophic Accumulation Of Proteins And The Antitumor Agent Smancs. Cancer Res.12, 6387-92 (1986).

5. Greish, K. Enhanced Permeability and Retention (EPR) Effect for Anticancer Nanomedicine Drug Targeting. Methods Mol Biol. 25-37 (2010). 
6. Walsby, AE. Gas Vesicles. Microbiol Rev. 58, 94-144 (1994).

7. Shapiro, M. et al. Biogenic Gas Nanostructures As Ultrasonic Molecular Reporters. Nat Nanotechnol. 9, 311-316 (2014).

8. Kunth, M., Lu, G., Witte, C., Shapiro, M., \& Schröder, L. Protein Nanostructures Produce Self-Adjusting Hyperpolarized Magnetic Resonance Imaging Contrast through Physical Gas Partitioning. ACS Nano. 12, 10939-10948 (2018).

9. Bharathiraja, S. et al. Chlorin e6 Conjugated Silica Nanoparticles for Targeted and Effective Photodynamic Therapy. Photodiagnosis Photodyn Ther. 19, 212-220 (2017).

10. Kostenich, G., Zhuravkin, I., Furmanchuk, A., \& Zhavrid, E. Photodynamic Therapy With Chlorin E6. A Morphologic Study Of Tumor Damage Efficiency In Experiment. J Photochem and Photobiol. 11, 307-318 (1991).

11. Chin, WW. et al. In-Vivo Optical Detection of Cancer Using Chlorin e6 Polyvinylpyrrolidone Induced Fluorescence Imaging and Spectroscopy. BMC Medical Imaging. 9 (2009).

12. Lakshmanan, A. et al. Preparation Of Biogenic Gas Vesicle Nanostructures For Use As Contrast Agents For Ultrasound And MRI. Nat Protoc. 12, 2050-2080 (2017).

13. Blaurock, A.E., Walsby, A.E. Crystalline Structure of the Gas Vesicle Wall from Anabaena Flos-Aquae. J Mol Biol, 105, 183-199 (1976).

14. Strunk, T. et al. Structural Model of the Gas Vesicle Protein GvpA and Analysis of GvpA Mutants in Vivo. Mol Microbiol. 81, 56-68 (2011).

15. Antonenko, Y. et al. Photodynamic Activity Of The Boronated Chlorin E6 Amide In Artificial And Cellular Membranes. Biochim Biophys Acta-Biomembr. 1838, 793-801 (2014).

16. Shton, I., Sarnatskaya, V., Prokopenko, L., \& Gamaleia, N. Chlorin e6 Combined With Albumin Nanoparticles As A Potential Composite Photosensitizer For Photodynamic Therapy Of Tumors. Exp Oncol. 37, 250-254 (2015).

17. Master, A., Livingston, M., \& Sen Gupta, A. Photodynamic Nanomedicine in the Treatment of Solid Tumors: Perspectives and Challenges. J Control Release, 168, 88-102 (2013).

18. Kostryukova, L., Prozorovskiy, V., Medvedeva, N., \& Ipatova, O. Comparison Of A New Nanoform Of The Photosensitizer Chlorin e6, Based On Plant Phospholipids, With Its Free Form. FEBS Open Bio. 8, 201-210 (2018). 
19. Zhao, L. et al. Efficient Delivery Of Chlorin E6 Into Ovarian Cancer Cells With Octalysine Conjugated Superparamagnetic Iron Oxide Nanoparticles For Effective Photodynamic Therapy. J Mater Chem B. 4, 7741-7748 (2016).

20. Yaghini, E. et al. Endolysosomal Targeting of a Clinical Chlorin Photosensitiser for Light-Triggered Delivery of Nano-Sized Medicines. Sci Rep. 7, (2017).

21. Kumari, P., Rompicharla, S., Bhatt, H., Ghosh, B., \& Biswas, S. Development Of Chlorin E6-Conjugated Poly(Ethylene Glycol)-Poly(D,L-Lactide) Nanoparticles For Photodynamic Therapy. Nanomedicine. 14, 819-834 (2019).

22. Jeong, H. et al. Photosensitizer-Conjugated Human Serum Albumin Nanoparticles for Effective Photodynamic Therapy. Theranostics 1, 230-23 (2011).

23. Umemura, S., Yumita, N., Nishigaki, R., \& Umemura, K. Mechanism Of Cell Damage By Ultrasound In Combination With Hematoporphyrin. Jpn J Cancer Res. 81, 962-966 (1990).

24. Zhang, P. et al. Iron Oxide Nanoparticles As Nanocarriers To Improve Chlorin E6Based Sonosensitivity In Sonodynamic Therapy. Drug Des Devel Ther. 12, 4207-4216 (2018).

25. Kimura, H. et al. Toxicity and Roles of Reactive Oxygen Species. Curr Drug Target Inflamm Allergy. 4, 489-495 (2005).

26. Sakharov, D. V., Elstak, E. D. R., Chernyak, B., \& Wirtz, K.W. A. Prolonged Lipid Oxidation After Photodynamic Treatment. Study With Oxidation-Sensitive Probe C11Bodipy 581/591. FEBS Lett. 579, 1255-1260 (2005).

27. Szokalska, A. et al. Proteasome Inhibition Potentiates Antitumor Effects Of Photodynamic Therapy In Mice Through Induction Of Endoplasmic Reticulum Stress And Unfolded Protein Response. Canc Res. 69, 4235-4243 (2009).

28. Schneider, C.A., Rasband, W.S., \& Eliceiri, K.W. NIH Image to ImageJ: 25 years of Image Analysis. Nature Methods. 9, 671-675 (2012).

29. Chen, X., Zhong, Z., Xu, Z., Chen, L., \& Wang, Y. 2',7'-Dichlorodihydrofluorescein as a Fluorescent Probe for Reactive Oxygen Species Measurement: Forty Years of Application and Controversy. Free Radic Res. 44, 587-604 (2010). 


\section{Acknowledgements}

We would like to thank Dr. Gang Zheng (University of Toronto) for critically reviewing this manuscript and Dr. Christine Allen (University of Toronto) for her support with this project. This study was supported by a grant from the Canadian Institutes of Health Research to J.G.

\section{Author Contributions}

A.F designed the experiments with advice from J.G. A.F. performed the experiments, analyzed the results, and prepared the manuscript. The manuscript was revised by J.G.

\section{Competing Interests}

The authors declare no competing financial interests. 
Table 1. Summary of size parameters for WT GVs and Ce6-GV. Results are shown as mean \pm S.D. (Image Fitting = 1 column)

\begin{tabular}{|l|l|}
\hline DLS Ce6-GV & $338 \pm 39 \mathrm{~nm}, \mathrm{n}=3$ \\
\hline DLS WT GV & $273 \pm 3 \mathrm{~nm}, \mathrm{n}=3$ \\
\hline TEM Ce6-GV & $\begin{array}{l}\text { Length: } 417 \pm 109 \mathrm{~nm}, \mathrm{n}=3 \\
\text { Width: } 196 \pm 38 \mathrm{~nm}, \mathrm{n}=3\end{array}$ \\
\hline TEM WT GV & $\begin{array}{l}\text { Length: } 379 \pm 23 \mathrm{~nm}, \mathrm{n}=3 \\
\text { Width: } 257 \pm 19 \mathrm{~nm}, \mathrm{n}=3\end{array}$ \\
\hline $\begin{array}{l}\text { Zeta Potential } \\
\text { Ce6-GV }\end{array}$ & $-30 \pm 4 \mathrm{mV}, \mathrm{n}=3$ \\
\hline $\begin{array}{l}\text { Zeta Potential } \\
\text { WT-GV }\end{array}$ & $-2.4 \pm 2 \mathrm{mV}, \mathrm{n}=3$ \\
\hline
\end{tabular}

Table 2. Comparison of $\mathrm{CD}_{50}$ values determined for MCF-7 cells and FaDuGFP cell lines following their exposure to free Ce6 and Ce6-GV. Molarity was calculated based on the molar concentration of Ce6. (Image Fitting = 1 column)

\begin{tabular}{|c|c|c|}
\hline & MCF-7 CD $50(M)$ & FaDu-GFP CD CD $(\mathrm{M})$ \\
\hline Ce6-GV & $2.6 \pm 1.4 \times 10^{-8}$ & $2.4 \pm 0.8 \times 10^{-8}$ \\
\hline Free Ce6 & $5.3 \pm 3.3 \times 10^{-6}$ & $5.5+4.0 \times 10^{-6}$ \\
\hline Free Ce6 $C_{50} /$ Ce6-GV CD 50 & 204 & 229 \\
\hline
\end{tabular}




\section{Figure Legends}

Fig. 1. A) Diagram depicting the conjugation of chlorin e6 (Ce6) carboxylic groups to the two lysine $\square$-amino groups (K19 and K59) of GvpA, the main protein component of Halobacterium sp. NRC-1 Gas Vesicles (GVs). B) GVs are air-filled protein nanobubbles recovered by flotation from lysed halophilic archaeabacteria. Purified floating layers are showed of wild type (WT) Halobacterium sp. NRC-1 GVs (white) and Ce6-modified GVs (green-grey color). (Image Fitting = 1 column)

Fig. 2. Characterization of WT GVs and Ce6-labeled GVs. Transmission electron micrographs of A) WT GVs and B) Ce6-labeled GVs indicating that modifying their surface with $\mathrm{Ce} 6$ yields comparable nanostructures. Similar dynamic Light Scattering size distribution profiles are observed for C) WT GVs and D) Ce6GVs. (Image Fitting = 1.5 column)

Fig. 3. The fluorescence spectrum for $\mathrm{Ce} 6$ released from acid-hydrolyzed Ce6GVs was comparable to that recorded for free Ce6. The emission spectra were recorded between 600 and $700 \mathrm{~nm}$ ( $\square$ exc $400 \mathrm{~nm}$ ) at $1 \mathrm{~nm}$ interval. The spectrum recorded for WT GVs hydrolysates confirmed the absence of a Ce6 spectral signature. All spectra are representative of recordings from two independent acid-hydrolyzed samples. (Image Fitting $=1$ column)

Fig. 4. Mean fluorescence intensities (MFI) as determined by fluorescenceactivated cell sorting (FACS) indicate that Ce6-GVs are internalized as a function of time by MCF-7 cells and FaDu-GFP cells at $37^{\circ} \mathrm{C}$ (Panels $\mathrm{B}$ and D). In contrast, minimal cellular uptake takes place at $4^{\circ} \mathrm{C}$ (Panels $A$ and $C$ ). Cells were equivalently treated with $670 \mathrm{nM}$ of $\mathrm{Ce} 6$ either as Ce6-GVs (closed circles) or as the free drug (closed triangles) over a 48-hour period. As projected, equivalent concentrations of WT GVs (closed squares) relative to Ce6-GVs did not generate significant MFI values. Results shown are representative of two independent trials performed in triplicate and presented as averaged MFI \pm S.D. $\square_{\text {exc }} 405$ nm; emission Filter 660-662 nm. (Image Fitting = 2 column)

Fig. 5. Merged confocal fluorescence and phase contrast microscopy images indicate that Ce6-GVs accumulate in the cytoplasm of cancer cells in a punctatelike pattern (in red) suggesting their entrapment in endocytic vesicles or vacuoles. The lack of co-localization of Ce6-GVs with the cell nucleus (Hoescht dye; blue stain) suggests that reactive oxygen species generated by Ce6-GVs being exposed to light would mainly accumulate in the cytoplasmic compartment of MCF-7 (panels A-D) or FaDu-GFP (panels E-H) cells after 8 hours of incubation. (Image Fitting $=1.5$ column) 
Fig. 6. Dose-dependent cell viability study of MCF-7 cells or FaDu-GFP cells treated with free $\mathrm{Ce} 6$ (closed triangles) or Ce6-GVs (closed circles) and exposed (Panels B and D) or not (Panels A and C) to light as determined using the WST-1 cell viability assay. Results are shown as mean \pm S.D. $n=3$ from 2 independent experiments, with each concentration performed in triplicate. (Image Fitting $=\mathbf{2}$ column)

Fig. 7. Production of Reactive Oxygen Species (ROS) in MCF-7 and FaDu-GFP cells treated with Ce6-GV, WT GV, or free Ce6. The ROS-sensitive probe DCFH-DA was subsequently loaded into treated cells with or without light activation of Ce6. ROS levels were quantified using a fluorescence plate reader. The results are shown as mean \pm S.D. $(n=10)$. Statistical significance was determined with a paired t-test, $p<0.05^{*}, p<0.01^{\star *}, p<0.001^{* *}, p<0.0001$ $\star \star \star *$. (Image Fitting $=1.5$ column)

a

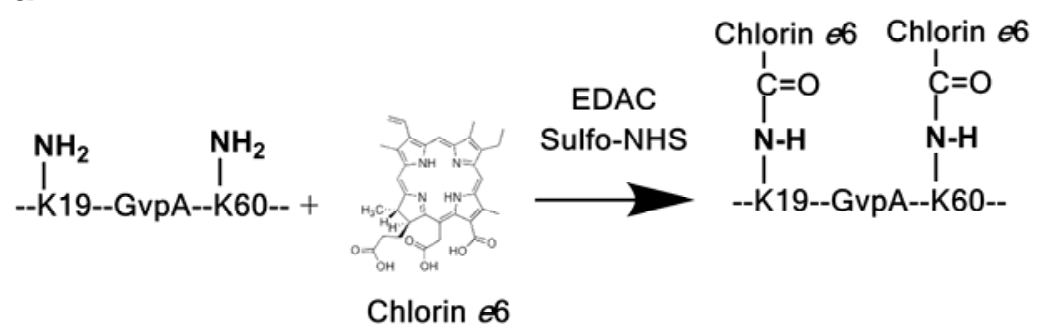

b

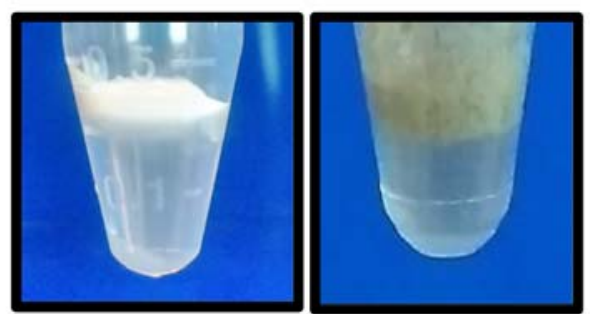

Fig. 1. 
bioRxiv preprint doi: https://doi org/10.1101/771881; this version posted September 19, 2019. The copyright holder for this preprint (which was not certified by peer review) is the author/funder, who has granted bioRxiv a license to display the preprint in perpetuity. It is made available under aCC-BY-ND 4.0 International license.

a

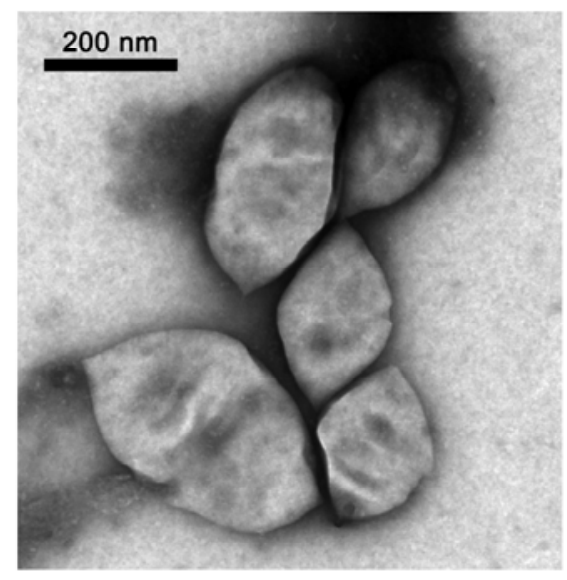

C

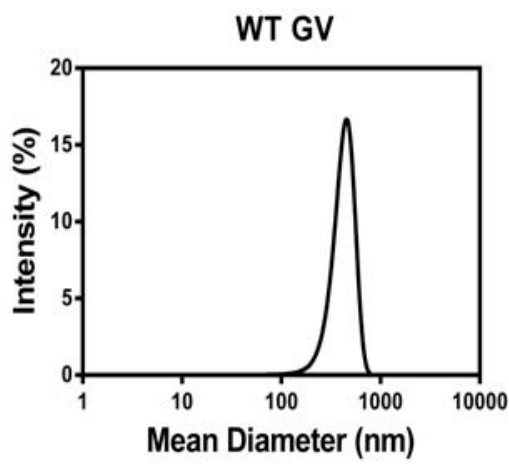

b

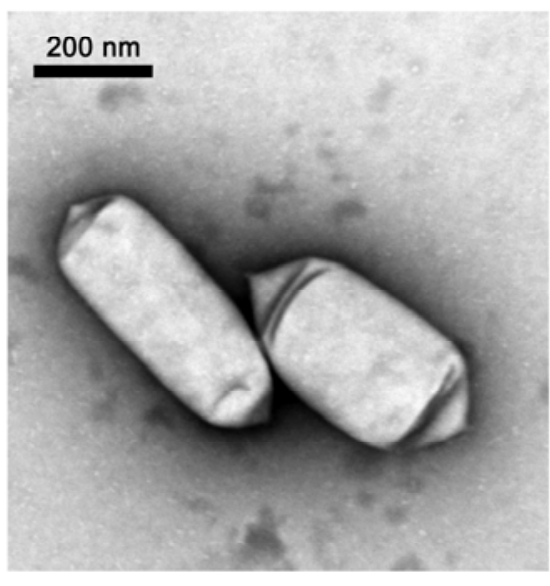

d

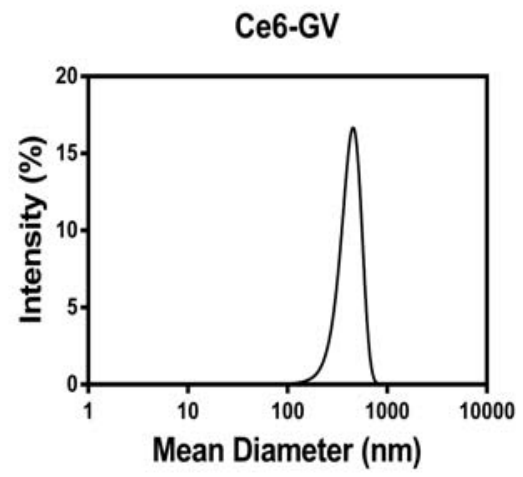

Fig. 2 . 
bioRxiv preprint doi: https://doi.org/10.1101/771881; this version posted September 19, 2019. The copyright holder for this preprint (which was not certified by peer review) is the author/funder, who has granted bioRxiv a license to display the preprint in perpetuity. It is made available under aCC-BY-ND 4.0 International license.

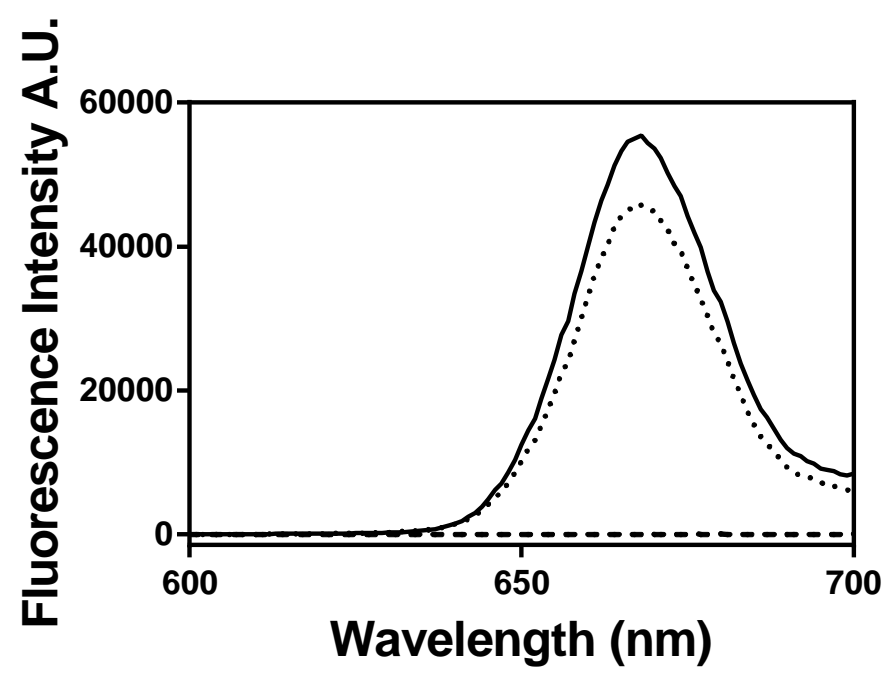

Fig. 3. 
a

$4{ }^{\circ} \mathrm{C}$ MCF-7

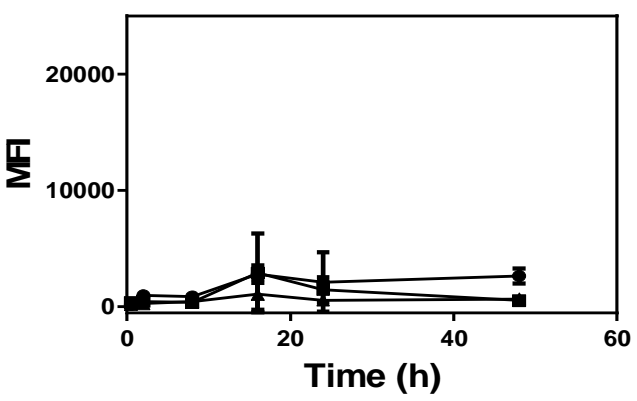

C

$4{ }^{\circ} \mathrm{C}$ FaDu-GFP

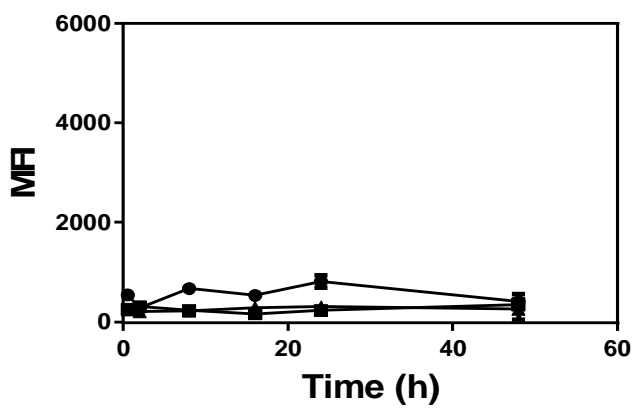

b

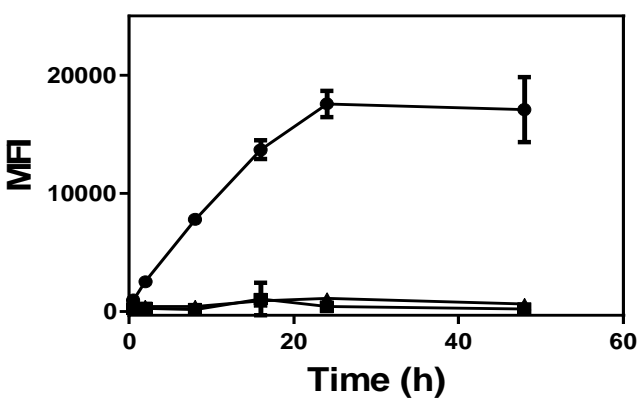

d

$37^{\circ} \mathrm{C}$ FaDu-GFP

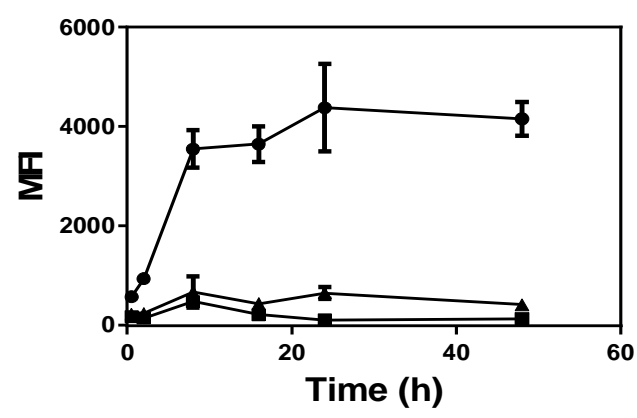

Fig. 4. 
bioRxiv preprint doi: https://doi.org/10.1101/771881; this version posted September 19, 2019. The copyright holder for this preprint (which was not certified by peer review) is the author/funder, who has granted bioRxiv a license to display the preprint in perpetuity. It is made available under aCC-BY-ND 4.0 International license.
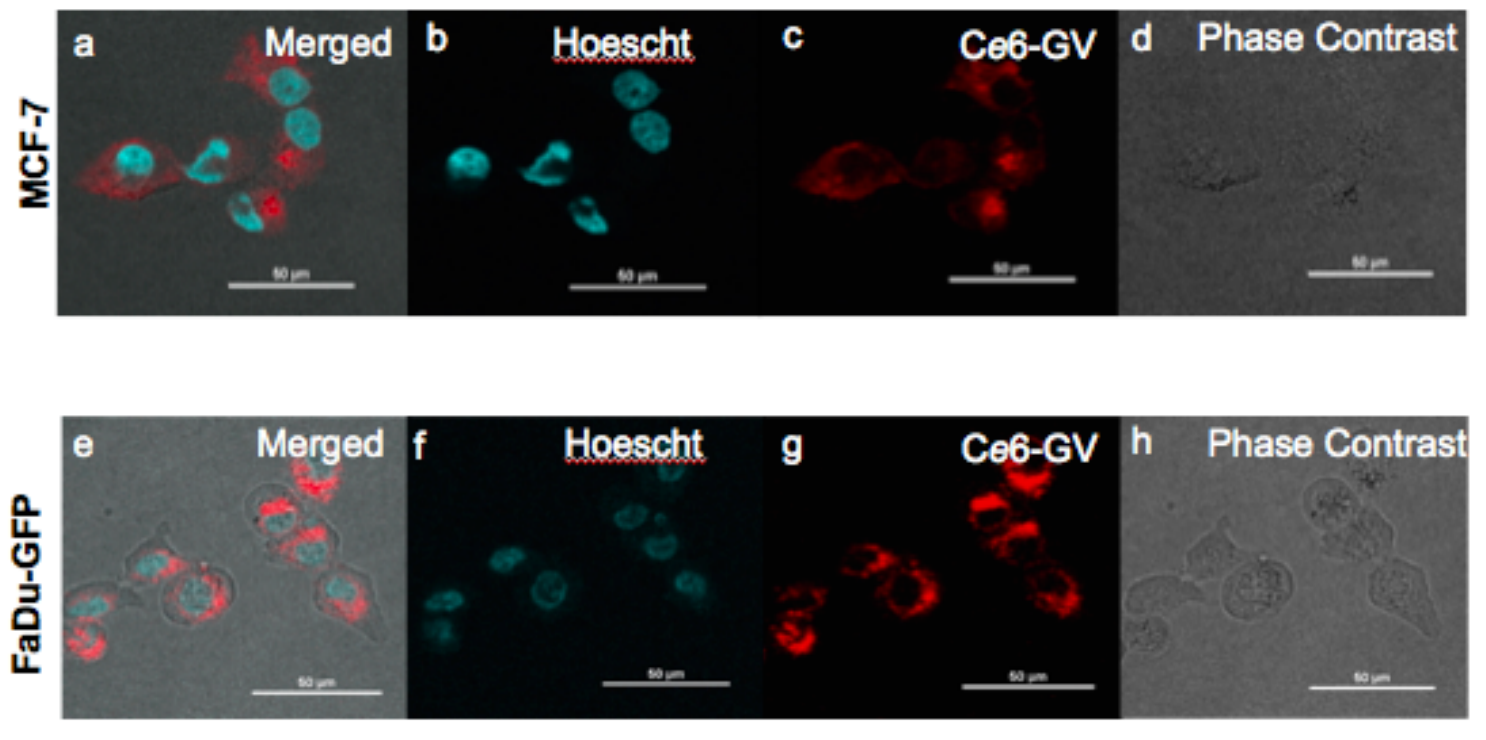

Fig. 5. 
a

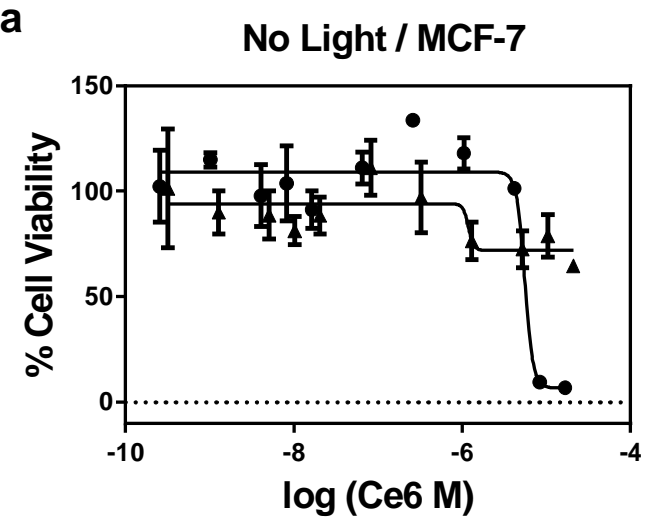

C

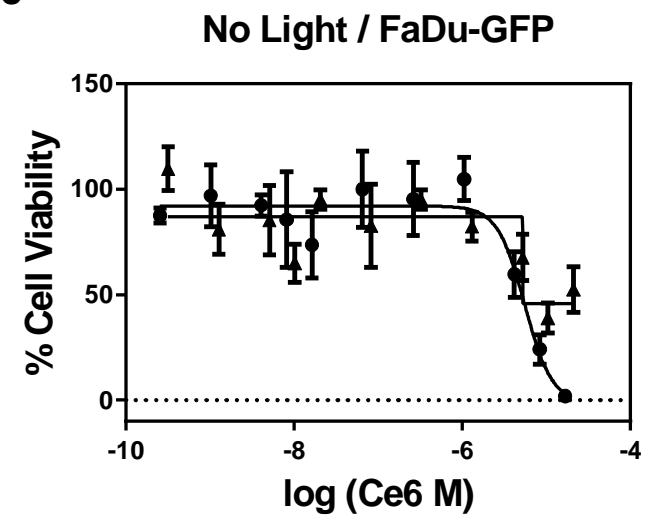

b

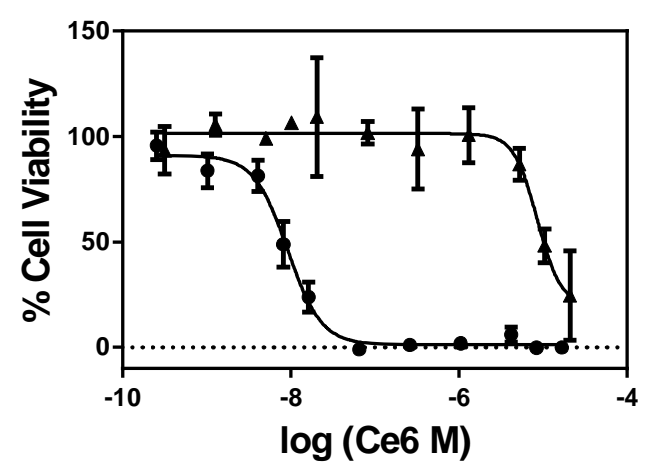

d

\section{Light / FaDu-GFP}

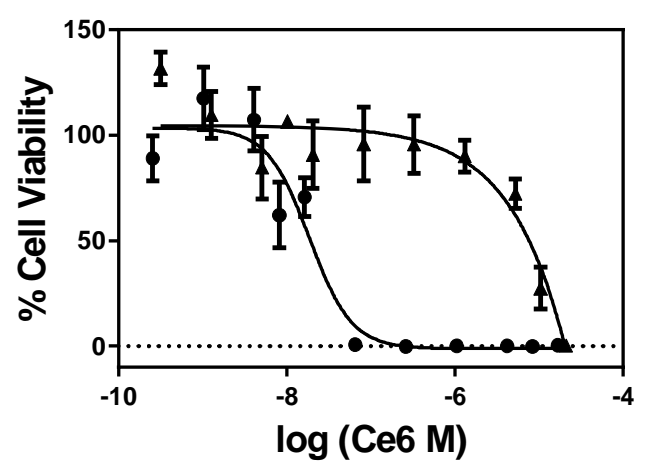

Fig. 6. 
a

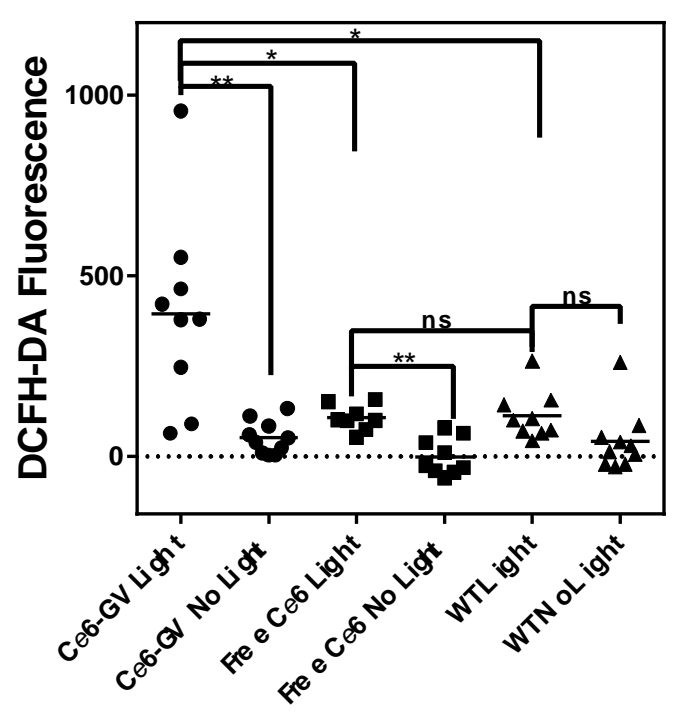

b

FaDu-GFP

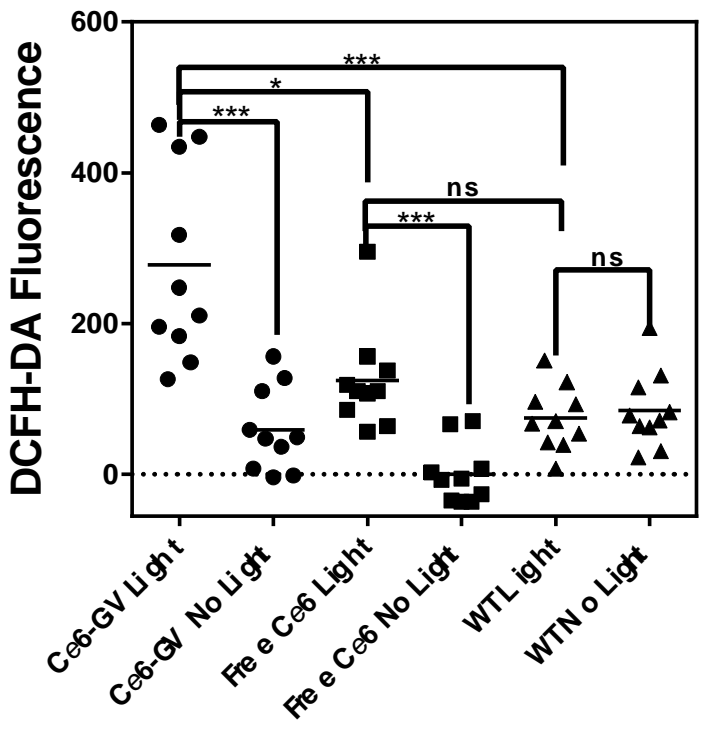

Fig. 7. 